\title{
Radio Frequency (RF)-based, Real-Time, Indoor Localization System for Unmanned Aerial Vehicles and Mobile Robot Applications
}

\author{
Sándor Tihamér Brassai, István Zsolt Székely \\ Sapientia Hungarian University of Transylvania, Department of Electrical \\ Engineering, Corunca 1.C, 540485, Romania, e-mail: tiha@ms.sapientia.ro, \\ szekely.istvan@ms.sapientia.ro
}

\begin{abstract}
The goal of this paper is to describe the indoor localization of a moving object, such as, an UAV or mobile robot, in real time. For localization, the triangulation method is used, based on a multiple fixed points anchors. The distance measurement between the moving object and the anchors is realized with DWM1000 UWB compliant, wireless transceiver modules. For distance measurement, the double-sided, two-way ranging method is used. The position is revealed after the triangulation is corrected, based on the Kalman filter. This computationally intensive task is running on FPGA and provides a realtime operation. One of the anchors is connected to FPGA with a serial communication interface. For the triangulation and Kalman filter, hardware module generation high level synthesis, is used. In the paper, in addition to the implementation, measurement results are also presented for real-time localization.
\end{abstract}

Keywords: Indoor localization; FPGA; High level synthesis; Kalman filter

\section{Introduction}

Indoor localization is a timely, active research area, with more challenges to be solved. More and more indoor applications require precise localization: mobile vehicle applications in factories and mines, healthcare applications [1], [2], fitness monitoring, assisted living [3], technical lab applications, games and entertainment, building automation [4] [5].

The reliability, required accuracy and response time, determine the selection of a solution/technology for the indoor localization implementation. The indoor positioning technologies can be grouped in several categories: wave propagation, image based and inertial navigation-based localization [5].

Other researchers categorize the indoor positioning technologies based on the kind of the signal used for object position determination: Radio Frequency Signals 
(RF), light, sound, audible and ultrasonic respective magnetic field-based solutions [6]. A wide variety of RF signal localization systems are known like WiFi, Bluetooth, ZigBee, RFID Ultra-Wideband. UWB technology presents some advantages in indoor positioning such as precision of time-of-flight measurement, multipath immunity and low power requirements [7] [8] [9].

Based on multiple reference devices, of which location is known, the position of the device attached to the moving object can be calculated. For distance estimation a variety of measurements can be used such as Received Signal Strength (RSS) [10] [11], Time of Arrival (ToA), Time Difference of Arrival (TDoA), and Angle of Arrival (AoA) [12].

Localization of moving objects in real time is a challenge, complex calculations are required. The emphasis and novelty of the paper is the acceleration of calculations of localization algorithm on the FPGA circuit. Critical parts of the real time localization process, for which calculations must be performed in a short period of time, such as triangulation, pre-filtering and the Kalman Filter, are modeled and implemented in hardware. For the future, the aim is to integrate into a single system the indoor localization methods, UAV systems [13] [14] and mobile robots [15] [16] [17], enabling mobile robots and UAV systems to be used for special indoors or even outdoors applications.

In this paper the chosen methods, algorithms, and their implementation are discussed. The paper is divided into six subsections followed by the conclusions, which summarize the results obtained. The indoor localization background is presented in section two. Theoretical background of the triangulation method and Kalman filter is detailed in the third and fourth sections. The fifth part describes the triangulation and Kalman filtering algorithms implemented on FPGA as well as the stand used to carry out measurements. The sixth section summarizes the results of the localization obtained with simulations and real measurements under laboratory conditions.

\section{Indoor Localization Background}

The project's aim is the development of a positioning system, used to locate the robot's position in real time.

There are many different localization systems and technologies which can be used for measuring an objects position. Each of these systems have their own advantages and disadvantages. Recent articles point out different aspects and applications of these, like, accuracy, energy efficiency, scalability, cost and such [18]. The goal was to use a system which is accurate and energy efficient, as well as it doesn't require any special or additional equipment. Recently UWB (ultrawideband modules) have become popular in localization, because of low energy 
consumption and accuracy. Using such modules, there were different attempts to develop better indoor localization systems, for either better accuracy or multiple tags, since these algorithms mostly rely on message transmission [19] [20]. The goal is to achieve an accurate position measurement using few moving objects, so an adequate hardware and algorithm are used and implemented.

The system using the triangulation technique measures the distance to different anchored points. For the distance measurement, DWM 1000 ultra-wide-band modules based on radio frequency were used. To improve the resulting position, after triangulation, a filtering algorithm is applied, to achieve a better estimation of the objects position. Both the triangulation and the filter algorithms are running in parallel on the FPGA.

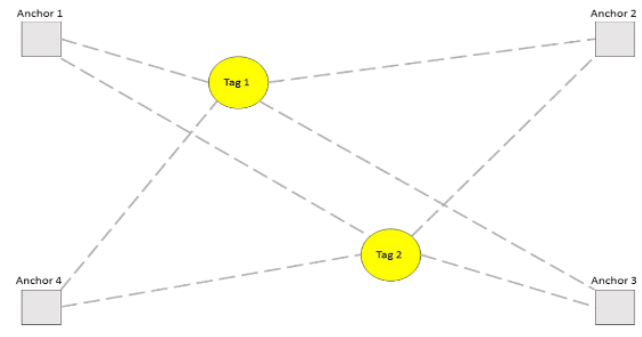

Figure 1

Essential connections

The RF modules used here, for the distance measurement, are a dedicated hardware, DWM1000, developed by DecaWave. The devices implied in position determination, are separated in two categories (Figure 1). In the first group there are the those with known and fixed positions and in the second group are the ones, in which the position has to be computed. These modules are deployed in the field, at least three in fixed positions and at least one is attached to the moving object, of which, the position has to be calculated.

The device controlling the RF modules, is an ESP8266 microcontroller. There is a written and working program library, for the measurements, which was rewritten according to the project's goals.

In the case of the moving target, of which, the position has to be measured, the ESP module is attached to the FPGA on SPI protocol (Figure 2). After measuring the distances, for noise reduction, a filter algorithm is used and then the location is computed using the triangulation method. The newly acquired location is optimized with a Kalman-filter. The filtering algorithms are also implemented in the FPGA circuit. 


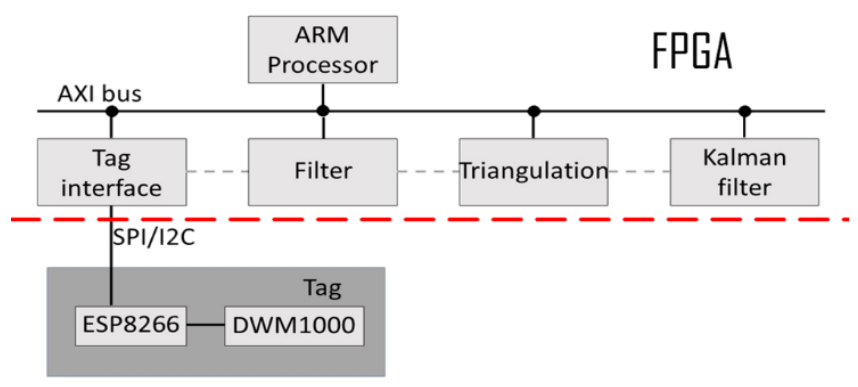

Figure 2

Block diagram of system architecture

\subsection{Distance Measuring Methods with RF Modules}

Choosing the right distance measuring method is crucial, in order to achieve accurate measurements. The primary target was to achieve an accuracy of $10 \mathrm{~cm}$, or less, with a data acquisition rate of $10 \mathrm{~Hz}$ or higher.

Using the radio frequency based modules, the following methods can be used to measure the distance: signal's strength, signal's angle of arrival, signal's time of arrival, and signal's differential time of arrival, and the alternatives of the above mentioned, like single-sided two-way ranging or double-sided two-way ranging.

Since the signal's strength significantly varies, while the movable target is stationary, this option was not viable. The signal's angle of arrival was not viable either, since in an environment, where there are many obstacles, the reflection of the signal from these objects makes it difficult to calculate the distance of the target. In addition, this method requires more anchors, in order to measure the distance with a high accuracy. The calculation of the time of arrival consists in the following: a device that wants to know its distance sends out a message at time T1. Another device receives this message at time T2, and by this, the flight time can be calculated, if their time is synchronized.

\subsubsection{Double-sided Two-Way Ranging}

The above-mentioned method requires an almost perfect synchronization, because even a very slight difference can cause serious measurement errors, $30 \mathrm{~cm} / 1 \mathrm{~ns}$. [21] This concept was modified to reduce the needs of the synchronization. The new concept was based on the difference of the arrival times. The first one was the one-sided, two-way ranging. In this scenario, the tag sends a message at time T1. The anchor receives it at time $\mathrm{T} 2$. The anchor sends back another message at time $\mathrm{T} 3$, which is received by the tag at time T4. In this way, there are four timestamps in total, two pointing to the time when the messages were sent, and two to the messages received. With these timestamps, the distance can be calculated, assuming that the distance between the two devices remains unchanged. 
tof $=\frac{(t 4-t 3)-(t 2-t 1)}{2}$

This was much better than the previous method, but still wasn't enough, so it was further developed. This method is called double-sided two-way ranging. This one is improved compared to the previous one by inserting an additional message, with this there are 6 timestamps in total, with which the distance can be measured [22].

$t o f=\frac{(t 4-t 1) *(t 6-t 3)-(t 3-t 2) *(t 5-t 4)}{(t 4-t 1)+(t 6-t 3)+(t 3-t 2)+(t 5-t 4)}$

There is a drawback, when more messages are exchanged, the accuracy improvement is less and less and when using multiple messages for range calculation, the accuracy can be even worse, if the target is moving [21].

\subsection{Measuring Distance with an Anchor and a Tag}

In the program library an example application of double-sided two-way ranging between two modules was written already. The tag is initiating the distance measurement by sending a poll message, the anchor receives the message, acknowledges it, after which the tag sends all the necessary timestamps for the calculation of the distance. After that the anchor sends back a message with the calculated distance and both sides know the result. This example had a small problem, which appeared in the interruption of the communication after random amounts of time, so it was corrected accordingly.

\subsubsection{Results Achieved by Others}

The DecaWave manufacturer states that the used DWM1000 modules, can achieve an accuracy of $10 \mathrm{~cm}$ indoors [23]. Most of the users of this module achieved an accuracy of $5 \mathrm{~cm}$; in some cases, they even achieved $2 \mathrm{~cm}$. Examining the measured and the real distances, it is clear that every module has an offset which is usually between 20 to $30 \mathrm{~cm}$. One of the best measurements others have achieved was with a $23.6 \mathrm{~cm}$ offset and a $2 \mathrm{~cm}$ standard deviation. There were cases, where the measurements were quite diverse.

This time they realized that the configuration used for the distance measurement was affecting it greatly, since these modules have different options for different use-cases. From measurement results presented in other works can be seen that at close range, and/or having different objects in the environment between the two modules, different distance values will be measured (Table 1). 
Table 1

Test results provided by Steven (https://github.com/thotro/24ocaliz-dw1000/issues/205)

\begin{tabular}{|l|l|l|l|}
\hline $\begin{array}{c}\text { Real distance } \\
{[\mathrm{cm}]}\end{array}$ & $\begin{array}{l}\text { Measured } \\
\text { distance }[\mathrm{cm}]\end{array}$ & $\begin{array}{l}\text { Average } \\
\text { error }[\mathrm{cm}]\end{array}$ & $\begin{array}{l}\text { Standard } \\
\text { deviation } \\
{[\mathrm{cm}]}\end{array}$ \\
\hline 25 & 18.2 & -6.8 & 2.030 \\
\hline 50 & 42.1 & -7.9 & 1.901 \\
\hline 100 & 101.4 & 1.4 & 2.063 \\
\hline 150 & 161.2 & 11.2 & 1.454 \\
\hline
\end{tabular}

\subsubsection{Results}

There were varied results acquired during the measurements. The settings of the modules and the environment in which the measurements were made, affected the results. These modules can be configured for a certain goal. The performance of the module was set to the maximum, which means that the data rate and pulse frequency is set to the highest level. The maximum range was also reduced, at which the measured distance was reduced. Different measurement results showed that the modules needed calibration.
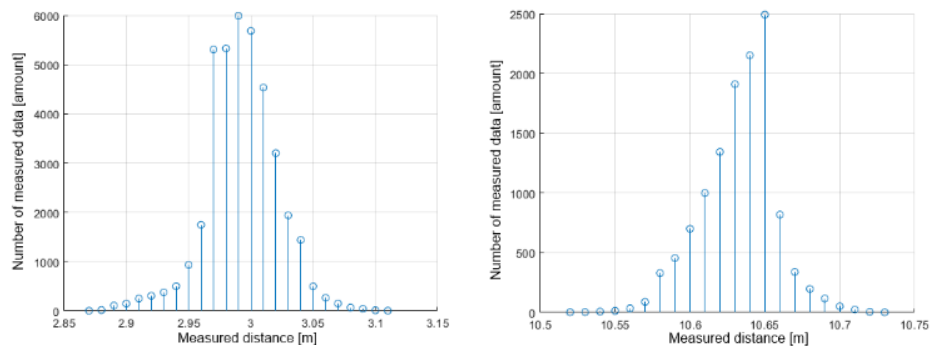

Figure 3

Test results: real distance $2.5 \mathrm{~m}$, offset $50 \mathrm{~cm}$, deviation $5 \mathrm{~cm}$, Test results: real distance $10 \mathrm{~m}$, offset $60 \mathrm{~cm}$, deviation $5 \mathrm{~cm}$

When only one anchor and one tag was used, the offset was usually around $40 \mathrm{~cm}$. The deviations of our measurements were not greater than $10 \mathrm{~cm}$ (Figure 3). It has to be mentioned, that in case of measurement with multiple anchors, the accuracy decreases and problems occur in the communication between the modules presenting in the random shutdown of communications.

\section{Triangulation Method}

The triangulation method is used to find the position of a target, typically in a twoor three-dimensional space. The method is based on at least three points (positions are known) and with the distance of the target to these points, the device's exact 
position can be calculated. For example, if the chosen space, where the positioning is used is two-dimensional, with one fixed point, a circle around the anchor, on which the target might be, can be determined. By using another fixed point, the section of the two circles give two points, where the target can be. If the exact position is needed, and both options are acceptable, then another anchor is needed. By this, another one of the points is eliminated, and the position of the device is exact. In three-dimensional space, it is slightly different. The only difference between the triangulation in two- or three-dimensional space is that the distance calculated results in a sphere. If two spheres are intersected, then they will provide a circle, which is similar to the previous one. By this, it can be said that increasing by one the number of dimensions results in an increase by one of the necessary fixed points to calculate the exact position. With this method, generally, in an $\mathrm{N}$ dimensional space, $\mathrm{N}+1$ reference points are needed. If the triangulation method is used in a three-dimensional space, and all the reference points are on the lowest possible position, then the only position, where the unknown device's location can theoretically be, is somewhere above the plane created by the anchors. In this case, the second point is automatically discarded. If the device can be on both sides of the plane, $\mathrm{N}$ amount of reference points will not be enough. If four anchors are used in space, then another question must be taken into consideration. If all four anchors are on the same plane, then the exact position cannot be calculated, because the exact position is still unknown, since there are two possible positions. If the four anchors are not on the same plane, then one option of the two possible positions is going to be removed, since the distance of the fourth anchor is not the same for the two, it will match only one.

It is considered that there are $\mathrm{k}$ amounts of anchors in which the position is known. These coordinates are (x, y, z). $s$ is the tag's position, which is unknown, and its coordinates are $(x, y, z)$. The distance to the anchors can be calculated with the following formula:

$t_{\mathrm{i}}=\sqrt{\left(\mathrm{x}_{\mathrm{i}}-x\right)^{2}+\left(\mathrm{y}_{\mathrm{i}}-y\right)^{2}+\left(\mathrm{z}_{\mathrm{i}}-z\right)^{2}}$

In this formula there are three unknown values, where result cannot be calculated so easily, using analytic methods. Every measurement has an error, which has to be taken into account. Because of this, it is divided into two parts: the real value, and the error.

$t_{\mathrm{i}}=s_{\mathrm{i}}(x, y, z)+\varepsilon(x, y, z)$

The error is calculated so the evaluation can be corrected. In order to reduce the error, an iterative method is used.

$s_{\mathrm{i}}(x, y, z)=s_{\mathrm{i}}(\tilde{x}+\Delta x, \tilde{y}+\Delta y, \tilde{z}+\Delta z)$

In order to reach the lowest possible error, linear evaluation is used, which consists in the following steps: in the first stage, each error value is defined. 
$s_{\mathrm{i}}(\tilde{x}+\Delta x, \tilde{y}+\Delta y, \tilde{z}+\Delta z)=s_{\mathrm{i}}(\tilde{x}, \tilde{y}, \tilde{z})+\frac{\partial s_{\mathrm{i}}}{\partial x} \Delta x+\frac{\partial s_{\mathrm{i}}}{\partial y} \Delta y+\frac{\partial s_{\mathrm{i}}}{\partial z} \Delta z$

After this, the partial derivatives are calculated.

$\frac{\partial s_{\mathrm{i}}}{\partial \tilde{x}}=\frac{-\mathrm{x}_{\mathrm{i}}+\tilde{x}}{\sqrt{\left(\mathrm{x}_{\mathrm{i}}-x\right)^{2}+\left(\mathrm{y}_{\mathrm{i}}-y\right)^{2}+\left(\mathrm{z}_{\mathrm{i}}-z\right)^{2}}}=a_{\mathrm{i}}$

$\frac{\partial s_{\mathrm{i}}}{\partial \tilde{y}}=\frac{-\mathrm{y}_{\mathrm{i}}+\tilde{y}}{\sqrt{\left(\mathrm{x}_{\mathrm{i}}-x\right)^{2}+\left(\mathrm{y}_{\mathrm{i}}-y\right)^{2}+\left(\mathrm{z}_{\mathrm{i}}-z\right)^{2}}}=b_{\mathrm{i}}$

$\frac{\partial s_{\mathrm{i}}}{\partial \tilde{z}}=\frac{-\mathrm{z}_{\mathrm{i}}+\tilde{z}}{\sqrt{\left(\mathrm{x}_{\mathrm{i}}-x\right)^{2}+\left(\mathrm{y}_{\mathrm{i}}-y\right)^{2}+\left(\mathrm{z}_{\mathrm{i}}-z\right)^{2}}}=c_{\mathrm{i}}$

Having them renamed is easier to use in the future.

$s_{\mathrm{i}}(\tilde{x}+\Delta x, \tilde{y}+\Delta y, \tilde{z}+\Delta z)=\mathrm{s}_{\mathrm{i}}(\tilde{x}, \tilde{y}, \tilde{z})+a_{\mathrm{i}} \Delta x+b_{\mathrm{i}} \Delta y+c_{\mathrm{i}} \Delta z$

From the equation the evaluated part was removed and vector remains for each anchor, holding the errors.

$\Delta s_{\mathrm{i}}=a_{\mathrm{i}} \Delta x+b_{\mathrm{i}} \Delta y+c_{\mathrm{i}} \Delta z$

The error is calculated using the measured and the evaluated distances difference.

The evaluated distance can be measured using the formula (1).

$\Delta s_{\mathrm{i}}=s_{\mathrm{i}}-\tilde{s}_{\mathrm{i}}$

After this, the formula (11) is rewritten into a matrix form, where the error vector $\Delta \ldots$ is $\boldsymbol{b}$, the partial derivatives' values $a_{\mathrm{i}}, b_{\mathrm{i}}, c_{\mathrm{i}}$ go into matrix $\boldsymbol{A}$, and the $\Delta x, \Delta y, \Delta z$ vector to $g$. Vector $g$ results are used to correct the evaluated position.

$\boldsymbol{b}=\boldsymbol{A g}$

Calculating $g$ from the formula above results in the following equation:

$g=A^{-1} b$

In many cases, the system is overdetermined, and because of this, the least square algorithm is used. First, the residual vector is calculated.

$\boldsymbol{r}=\boldsymbol{b}-\boldsymbol{A g}$

Its Euclidean square is used in order to be able to calculate vector $g$.

$\|\boldsymbol{r}\|^{2}=\boldsymbol{r}^{\mathrm{T}} \boldsymbol{r}=(\boldsymbol{b}-\boldsymbol{A g})^{\mathrm{T}}(\boldsymbol{b}-\boldsymbol{A g})$

$\|\boldsymbol{r}\|^{2}=\boldsymbol{b}^{\mathrm{T}} \boldsymbol{b}-2 \boldsymbol{g}^{\mathrm{T}} \boldsymbol{A}^{\mathrm{T}} \boldsymbol{b}+\boldsymbol{g}^{\mathrm{T}} \boldsymbol{A}^{\mathrm{T}} \boldsymbol{A} \boldsymbol{g}$

The result is equal to zero, and after that, $g$ is placed on one side and the rest to the other.

$-2 \boldsymbol{g}^{\mathrm{T}} \boldsymbol{A}^{\mathrm{T}} \boldsymbol{b}+\boldsymbol{g}^{\mathrm{T}} \boldsymbol{A}^{\mathrm{T}} \boldsymbol{A} \boldsymbol{g}=0$

From the resulted equation, the $\boldsymbol{b}^{\mathrm{T}} \boldsymbol{b}$ is removed, because the equation's derivative was used. 


$$
\boldsymbol{g}=\left(\boldsymbol{A}^{\mathrm{T}} \boldsymbol{A}\right)^{-1} \boldsymbol{A}^{\mathrm{T}} \boldsymbol{b}
$$

Finally, at this point, the vector $\boldsymbol{g}$ is calculated, and the evaluated coordinates can be improved.

$$
s=s+g
$$

This is an iterative method, so it is necessary to do this multiple times, until an error limit is reached, which is given by the user.

It is important to determine the application itself, the possibilities of placing the modules, and by this choosing the number of the reference points [24], [25]. The triangulation method was implemented in the FPGA, because the ESP8266 microcontroller couldn't calculate the position in time.

\section{Kalman Filter}

The Kalman filter is a linear quadratic estimator which can provide an accurate estimation of states. Kalman filters are typically used for linear systems having Gaussian noise. This filter can estimate states from the data provided by multiple sensors, which measurements are noisy. The Kalman filter doesn't have to store older data values. Kalman filters have relatively simple form and require relatively small computational power. There are two matrices, the covariance matrix of measurement noise $(\mathbf{Q})$ and the covariance of the observation noise $(\mathbf{R})$, which are responsible for the estimation. The $\mathbf{Q}$ with dimension $9 \times 9$ and $\mathbf{R} 3 \times 3$ was initialized with identity matrix multiplied by constant values initialized form the Simulink. In choosing these $\mathbf{Q}$ and $\mathbf{R}$ parameter values, their ratio matters. With a $\mathrm{Q} / \mathrm{R}$ ratio of 1 for example, the estimated values change slowly, neglecting most of the noise, in case of slower systems. When the ratio is 10 , the estimated values change fast but with less noise suppression [26].

Position prediction based on the transition model:

$$
\begin{aligned}
& \widehat{\boldsymbol{x}}_{\mathrm{k} / \mathrm{k}-1}=\boldsymbol{\Phi}_{\mathrm{k} / \mathrm{k}-1} \widehat{\boldsymbol{x}}_{\mathrm{k}-1} \\
& \boldsymbol{P}_{\mathrm{k} / \mathrm{k}-1}=\boldsymbol{\Phi}_{\mathrm{k} / \mathrm{k}-1} \boldsymbol{P}_{\mathrm{k}-1} \boldsymbol{\Phi}_{\mathrm{k} / \mathrm{k}-1}^{\mathrm{T}}+\mathbf{Q}_{\mathrm{k}-1} \\
& \hat{\mathbf{z}}_{\mathrm{k} / \mathrm{k}-1}=\mathbf{H}_{\mathrm{k}} \widehat{\boldsymbol{x}}_{\mathrm{k}-1}
\end{aligned}
$$

Update steps

$$
\begin{aligned}
& \boldsymbol{z}_{\mathrm{k}}=\left[x_{\mathrm{k}}, y_{\mathrm{k}}, z_{\mathrm{k}}\right]^{\mathrm{T}} \\
& \boldsymbol{\varepsilon}_{\mathrm{k}}=\boldsymbol{z}_{\mathrm{k}}-\widehat{\mathbf{z}}_{\mathrm{k} / \mathrm{k}-1} \\
& \widehat{\boldsymbol{x}}_{\mathrm{k}}=\widehat{\boldsymbol{x}}_{\mathrm{k}-1}+\boldsymbol{K}_{\mathrm{k}-1} \boldsymbol{\varepsilon}_{\mathrm{k}-1}
\end{aligned}
$$




$$
\begin{aligned}
\boldsymbol{R}_{\mathrm{k}} & =\boldsymbol{R}_{\mathrm{k}-1}+\boldsymbol{\varepsilon}_{\mathrm{k}} \boldsymbol{\varepsilon}_{\mathrm{k}}^{\mathrm{T}}-\mathbf{H}_{\mathrm{k}} \boldsymbol{P}_{\mathrm{k}} \mathbf{H}_{\mathrm{k}}^{\mathrm{T}} \\
\boldsymbol{K}_{\mathrm{k}} & =\boldsymbol{P}_{\mathrm{k} / \mathrm{k}-1} \mathbf{H}_{\mathrm{k}}^{\mathrm{T}}\left[\mathbf{H}_{\mathrm{k}} \boldsymbol{P}_{\mathrm{k}} \mathbf{H}_{\mathrm{k}}^{\mathrm{T}}+\boldsymbol{R}_{\mathrm{k}}\right]^{-1} \\
\boldsymbol{P}_{\mathrm{k}} & =\left(\mathrm{I}-\boldsymbol{K}_{\mathrm{k}} \mathbf{H}_{\mathrm{k}}\right) \boldsymbol{P}_{\mathrm{k} / \mathrm{k}-1}\left(\mathrm{I}-\boldsymbol{K}_{\mathrm{k}} \mathbf{H}_{\mathrm{k}}\right)^{\mathrm{T}}+\boldsymbol{K}_{\mathrm{k}} \boldsymbol{R}_{\mathrm{k}} \boldsymbol{K}_{\mathrm{k}}^{\mathrm{T}} \\
\boldsymbol{\Phi} & =\left[\begin{array}{ccccccccc}
1 & \mathrm{~T} & \mathrm{~T}^{2} / 2 & 0 & 0 & 0 & 0 & 0 & 0 \\
0 & 1 & \mathrm{~T} & 0 & 0 & 0 & 0 & 0 & 0 \\
0 & 0 & 1 & 0 & 0 & 0 & 0 & 0 & 0 \\
0 & 0 & 0 & 1 & \mathrm{~T} & \mathrm{~T}^{2} / 2 & 0 & 0 & 0 \\
0 & 0 & 0 & 0 & 1 & \mathrm{~T} & 0 & 0 & 0 \\
0 & 0 & 0 & 0 & 0 & 1 & 0 & 0 & 0 \\
0 & 0 & 0 & 0 & 0 & 0 & 1 & \mathrm{~T} & \mathrm{~T}^{2} / 2 \\
0 & 0 & 0 & 0 & 0 & 0 & 0 & 1 & \mathrm{~T} \\
0 & 0 & 0 & 0 & 0 & 0 & 0 & 0 & 1
\end{array}\right]
\end{aligned}
$$

Where $\boldsymbol{x}_{\mathrm{k}}$ is the state variables, $\boldsymbol{\Phi}_{\mathrm{k}}$ system matrix, $\mathbf{H}_{\mathrm{k}}$ the observation model, $\boldsymbol{z}_{\mathrm{k}}$ the measured positions, $\boldsymbol{K}_{\mathrm{k}}$ Kalman gain, $\mathbf{Q}_{\mathrm{k}}$ the covariance of the measurement noise, $\boldsymbol{R}_{\mathrm{k}}$ the covariance of the observation noise, $\boldsymbol{P}_{\mathrm{k}}$ estimate covariance matrix, $\varepsilon_{\mathrm{k}}$-error between the measured and estimated positions. Finally, the estimated positions are obtained from $[x(1) x(4) x(7)]$.

Transition model is defined based on the motion equation along the coordinates eq. (29).

\section{Implementation}

\subsection{Distance Measurements between Tags and Anchors}

For distance measurement between tag and anchors the double sided two-way ranging measurement was used (Figure 4).

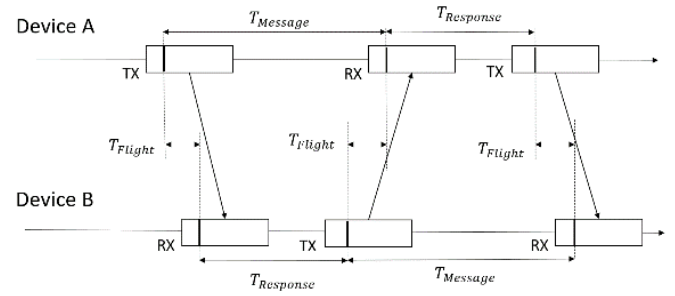

Figure 4

Double sided two-way ranging signal exchange between a tag and anchor 


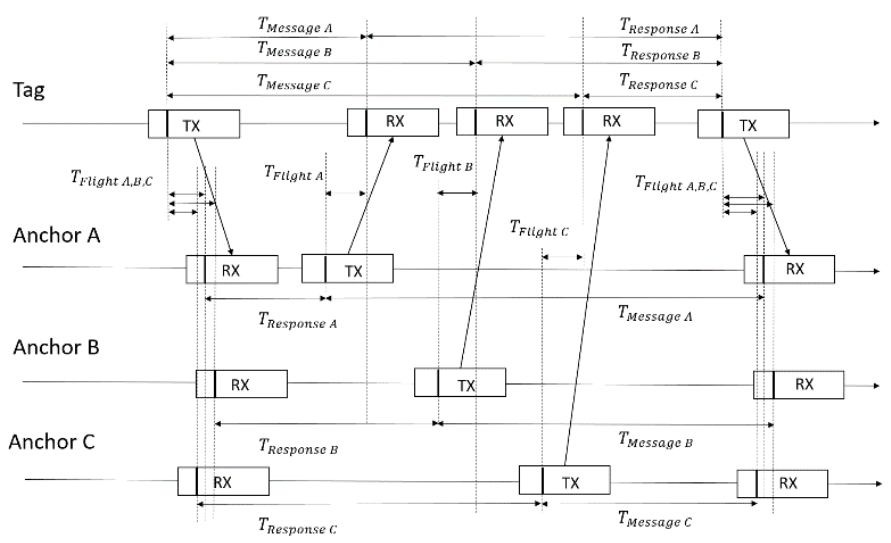

Figure 5

Double sided two-way ranging signal exchange between a tag and multiple anchors

By using multiple anchors, the communication between the tag and the anchors was modified, in order to achieve a faster distance measurement. In this case, the tag has sent one message to all of the anchors. After this, the anchors sent an answer for the polling message. These anchors sent the message one after another after a certain amount of time. After the tag received the messages from all the anchors, it sent another message containing the timestamps necessary to calculate the distance. After all the anchors received the message, each one calculates the distance, and sends back the results to the tag (Figure 5).

\subsection{Triangulation Method Implementation}

The triangulation hardware module was designed with high level synthesis in Vivado HLS environment. The function, from which the hardware module is generated, receives the position of the anchor devices as well as the distances to the fixed devices as inputs and provides the position of the device to be localized as output argument.

The positions of the fixed devices are stored in an array from which the triangulation module reads the values of the positions at the beginning of the calculation cycle. In the current configuration, the devices are spaced $2 \mathrm{~m}, 2 \mathrm{~m}$ and $2 \mathrm{~m}$ along the $\mathrm{X}, \mathrm{Y}$ and $\mathrm{Z}$ axes relative to the origin, and in the first phase of development were hardware coded, which meant many limitations during the tests. During the research we have modified many important parameters that can be given as inputs to the module.

The input and output arrays are partitioned to elementary signals. In addition to the output positions, the ap_valid protocol signals help synchronize the data transfer between the triangulation module and Kalman filter. The module's hardware resource requirements are summarized in Table 2. 
Table 2

Triangulation module hardware resource requirements

\begin{tabular}{|l|r|r|r|r|}
\hline \multicolumn{1}{|c|}{ Name } & BRAM_18K & DSP48E & FF & LUT \\
\hline DSP & - & - & - & - \\
\hline Expression & - & - & 0 & 1822 \\
\hline FIFO & - & - & - & - \\
\hline Instance & 2 & 22 & 5450 & 8853 \\
\hline Memory & 4 & - & 192 & 24 \\
\hline Multiplexer & - & - & - & 1082 \\
\hline Register & - & - & 1718 & - \\
\hline Total & 6 & 22 & 7360 & 11781 \\
\hline Available & 120 & 80 & 35200 & 17600 \\
\hline Utilization (\%) & 5 & 27 & 20 & 66 \\
\hline
\end{tabular}

\subsection{Kalman Filter Implementation}

The hardware for the Kalman filter algorithm was also generated by HLS synthesis. The function receives as input an array of $\mathrm{X}, \mathrm{Y}, \mathrm{Z}$ positions after the triangulation and also provides the estimated position coordinates in an array.

As for initial estimated values, a position of $\left[\begin{array}{lll}0 & 0 & 0\end{array}\right]$ is chosen for the tag, which is equivalent the Origin of the triangulation system. At the beginning, some time is necessary for the Kalman filter to reach the position of the real system. In this transitional state, the mobile system should be stationary.

Table 3

Kalman filter module hardware resource requirements

\begin{tabular}{|l|l|l|l|l|}
\hline \multicolumn{1}{|c|}{ Name } & BRAM_18K & DSP48E & FF & LUT \\
\hline DSP & - & - & - & - \\
\hline Expression & - & - & 0 & 180 \\
\hline FIFO & - & - & - & - \\
\hline Instance & 2 & 14 & 3043 & 4748 \\
\hline Memory & 16 & - & 0 & 0 \\
\hline Multiplexer & - & - & - & 1474 \\
\hline Register & - & - & 98 & - \\
\hline Total & 18 & 14 & 3141 & 6402 \\
\hline Available & 120 & 80 & 35200 & 17600 \\
\hline Utilization $(\%)$ & 15 & 17 & 8 & 36 \\
\hline
\end{tabular}

HLS_ARRAY_PARTITION forces the input and output arrays to be partitioned in hardware modules into individual inputs and outputs. In addition to the output filtered positions, the ap_valid protocol signal helps synchronize signal 
transmission between modules. The Kalman filter hardware resource requirements are summarized in Table 3.

Summing the hardware resources of triangulation and Kalman filter modules exceed the available hardware resources of Zynq XC7Z010, hardware resources are optimized during synthesis (Figure 6).

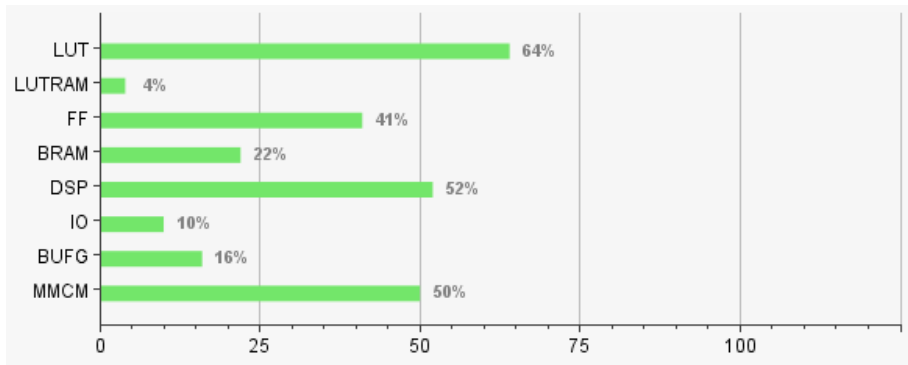

Figure 6

Full localization module hardware resource requirements

\subsection{One Degree of Freedom Arm for Tag Movement}

In order to test the localization system, measurements were made by mounting the moving device on a single-degree arm. The synchronization of the signals of the two measuring systems is easy to implement because both systems are integrated in the same FPGA circuit. Based on the position of the robotic arm and the estimated positions returned from the FPGA to the computer, the two measurements can be easily fitted.

The single-degree servo control loop consists of a cascade P and PID controller, a PWM signal generator, an H-bridge control circuit, and an encoder for decoding the servo angular position. The PWM signal generator, encoder and PID control are also implemented in FPGA circuits and are not described in detail since they are not present as the basis of the present research.

\subsection{Complementary Modules used for Tag Movement}

For measurements, the triangulation, Kalman Filter and single-degree positioning control modules are connected in the Xilinx System Generator (SG). Hardware co-simulation helped greatly in setting the correct parameters for the subunits and in developing a functional localization system. In the SG a multiple clock type system has been developed.

The data exchange over JTAG between the PC and the FPGA is synchronized to the clock signal of the JTAG interface, while the clock of the modules implemented in the FPGA is derived from the FPGA master clock. 
During the implementation of the hardware co-simulation-based design for the development board used, to derive the clock from the board master clock special attention must be paid.

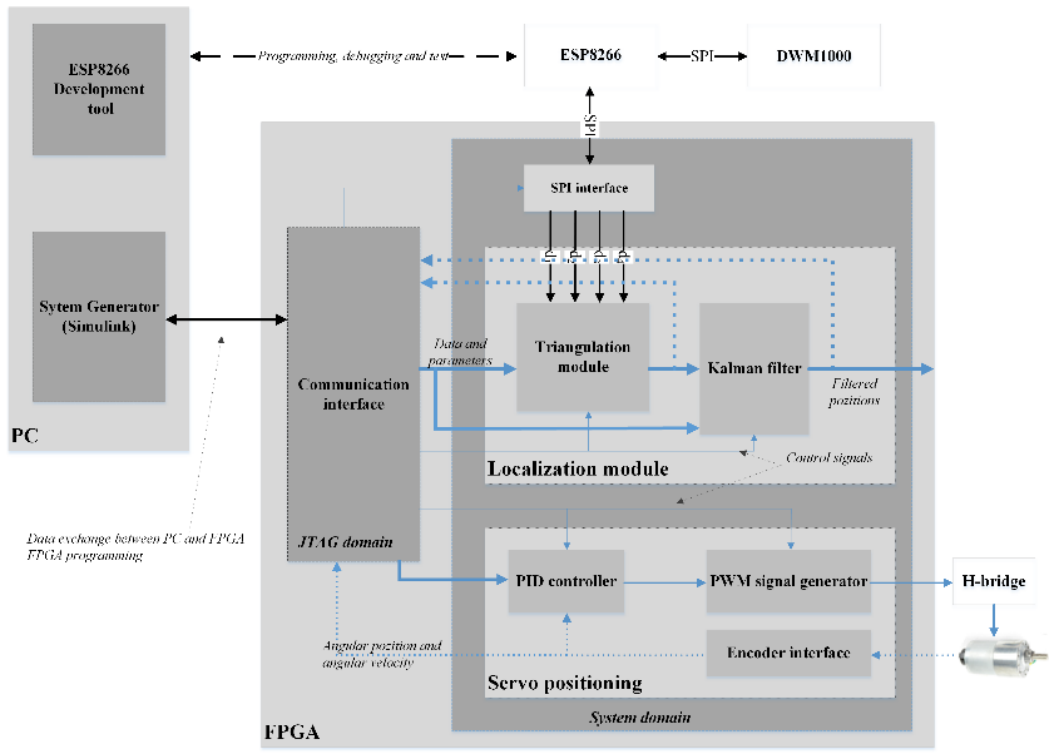

Figure 7

Hardware co-simulation system modules

set_property -dict \{ PACKAGE_PIN K17 IOSTANDARD LVCMOS33 \} [get_ports \{ sys_clock \}];

create_clock -add -name sys_clk_pin -period 10.00 -waveform $\{05\}$ [get_ports \{ sys_clock \}];

For the communication between the ESP and the FPGA a hardware implemented SPI protocol is used. The slave SPI, running in the FPGA, saves the distances in its own registers, which serve as inputs to the triangulation module.

The following diagram shows the connection of the modules.

Modules are parameterized from the Simulink model via the JTAG interface and partial results from the modules are also displayed or saved from the Simulink model.

The triangulation and Kalman Filter modules are exported from the Vivado HLS environment. The reference model, applied to the Kalman filter, based on which position estimation is realized, describes the motion equations along the three axes.

Based on the developed and tested model, an IP core is generated, which is fitted to the bus system of the FPGA based control unit of mobile robot and UAV. 


\section{Measurement Results}

\subsection{Test Results: Simulated -Inputs - Simulated Processing}

Depending on how many anchors are used, and which are the starting evaluated positions, different results were achieved. The calculations were made, while the device was stationary. First, four anchors were used (Figure 8), which were not located on a single plane. Different starting values were used, and the results were all the same. The data used for calculating the position was real.
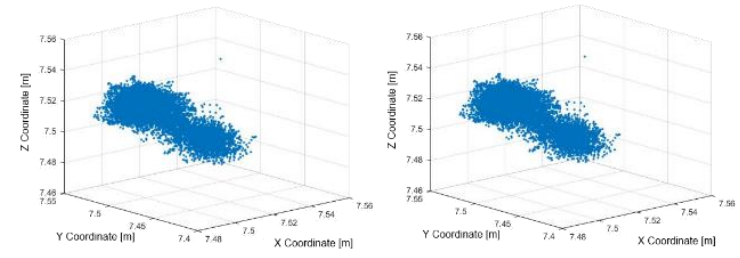

Figure 8

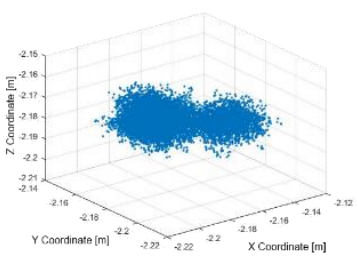

The result of the positioning using: four anchors, varying starting evaluated values; three anchors, starting evaluated values initialized with positive values; three anchors, starting evaluated values initialized with negative values

When three anchors were used (Figure 8) different starting evaluated values gave different results, using the same measurements as previously with four anchors. When positive values were used, then the evaluation was similar when measuring with four anchors.

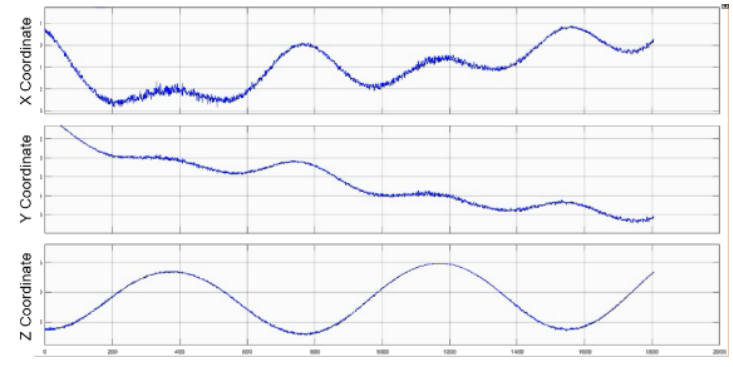

Figure 9

Result for triangulation method, which is running on the hardware module simulated on Simulink SG

However, when the starting evaluated values were negative, the triangulation method localizes the device on the other side of the plane, which is formed by the three anchors.

On Figure 9 a simulation is presented with generated data from Simulink. Multiple sine waves were generated with different parameters as distances in order to achieve these coordinates. On the first figure are the calculated coordinates received from the simulated hardware (Figure 10). 
For the Kalman filter testing, the same coordinates were used as in the triangulation methods testing. In the following two figures the $\mathrm{Q}$ and $\mathrm{R}$ ratio was changed in order to see their accuracy and speed of convergence (Figure 10).
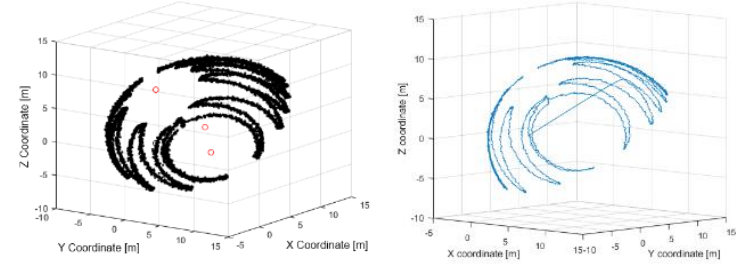

Figure 10

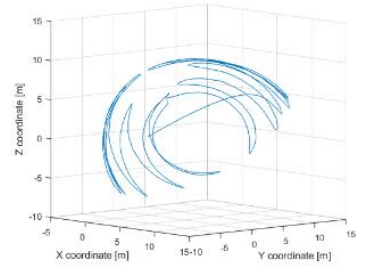

The results received from the hardware model simulated in Simulink SG. Results for triangulation, and Kalman estimation with two different parameter sets of $\mathrm{R}$ and $\mathrm{Q}$.

\subsection{Test Results: Simulated Inputs - Hardware Processing}

The following measurements show the results obtained by testing the triangulation and Kalman filter modules. The calculations were running on the FPGA circuit. The inputs from the Simulink environment were generated, according to a real task. Using a cylindrical, two-degree of freedom robotic arm, the input points were generated, from which the distances to the anchors were calculated. The calculated distances, to illustrate the real circumstance, were perturbed with a normal distribution noise (variance 0.01 and different sample time 0.008, 0.007, 0.009). The simulation sampling time was $1 \mathrm{~ms}$.

The robot arm was raised along the Z-axis and also was rotated along the Z-axis by +-360 degrees forth and back during lifting, as can be seen in Figure $12 \mathrm{~d}$. The following Figure 11 shows the measured results.

Along axis $\mathrm{X}, \mathrm{Y}, \mathrm{Z}$ the coordinates calculated by coordinates obtained with the Kalman filter for different Q parameter sets $(\mathrm{k}-1, \mathrm{k}-2, \mathrm{k} 3)$ is presented. During the simulations, the $\mathrm{R}$ matrix values along the main diagonal are set to 2 and the $\mathrm{Q}$ matrix values to $[0.0005,0.001,0.002]$ with sampling time $\mathrm{T}$ used in the model for prediction of [ [ $\left.\begin{array}{lll}0.01 & 0.010 .01\end{array}\right]$.

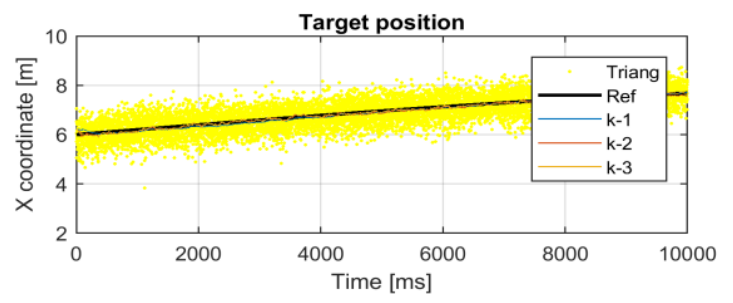



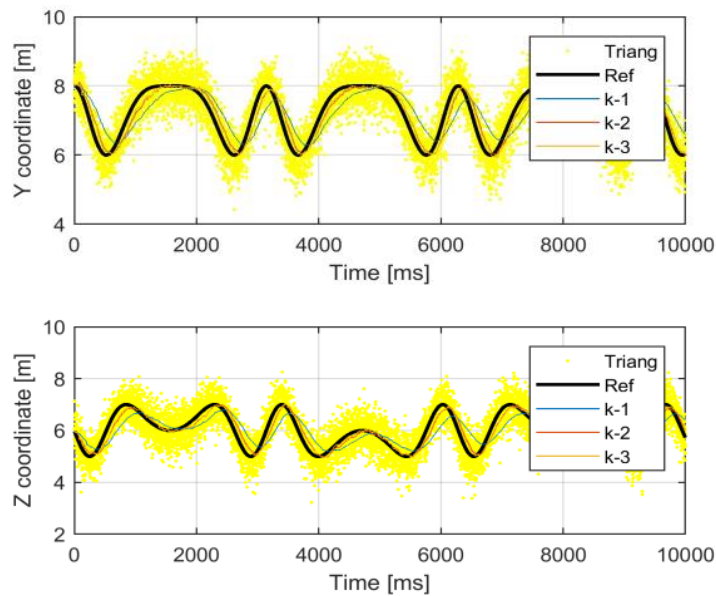

Figure 11

Simulated inputs, real processing of triangulation and Kalman filtering on FPGA for X, Y, Z axes. For each axis is presented the triangulation-based estimation (Triang), original reference signal (Ref), and results for Kalman filter for three parameter sets (k-1, k-2 and k-3)

The simulations support the correct operation of both the triangulation and Kalman filter modules. By tuning the Q parameter of the Kalman filter, can be found an appropriate mid-way solution that ensures the noise suppression with minimal error of approximation of original reference signal.
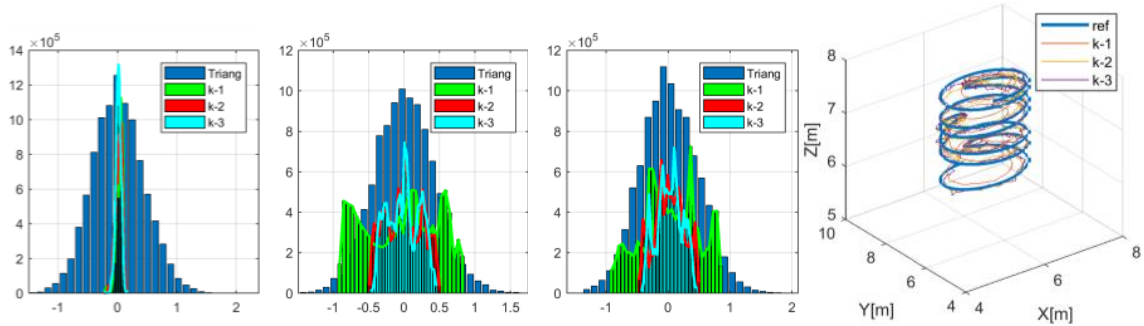

Figure 12

Error distribution along axes $\mathrm{X}, \mathrm{Y}$ and $\mathrm{Z}$ (from left to right) for triangulation-based estimation (Triang), and for Kalman filter for three parameter sets (k-1, k-2 and k-3). Representation of the path evolution in XYZ coordinate system for original reference signal (ref) and for Kalman filter for three parameter sets (k-1, k-2 and k-3)

In Figure 12 the distribution of the difference between the original signal, the distribution of error between the original signal and the estimation with the triangulation and the three variants of the Kalman filter is presented.

Comparing the results obtained by triangulation with the original coordinates, the calculated variances on the $\mathrm{X}, \mathrm{Y}$ and $\mathrm{Z}$ axes are $0.19,0.18$ and 0.21 respectively. The variance of the error between the original signal and the signals obtained for 
three different tuning sets of the Kalman filter is $0.0031,0.0032$ and 0.0021 respectively along the $\mathrm{X}$ axis, $0.2567,0.0664,0.0500$ along the $\mathrm{Y}$ axis, as well as, $0.2056,0.0579$ and 0.0423 , respectively along the $\mathrm{Z}$ axis.

Comparing the variation values for triangulation and Kalman filter, we can also deduce from this, but not only, the usefulness of the Kalman filter for noise reduction. The Figure $12 \mathrm{~d}$ illustrates the evolution in tridimensional space of the original path and for the three cases estimated by Kalman filter.

\subsection{Test Results: Real Inputs - Hardware Processing}

In real measurements three anchors were used. The tag was moved in space along a semi-circle using a dc servomotor. The servomotor's shaft was parallel to the $\mathrm{XZ}$ plane. The axis was tilted by 30 degrees relative to the $\mathrm{XY}$ plane, so changes can be seen on all three axis. The motor shaft position was $1.4 \mathrm{~m}, 2 \mathrm{~m}$ and $-10 \mathrm{~cm}$ relative to the $\mathrm{X}, \mathrm{Y}$ and $\mathrm{Z}$ axis respectively. The arm's length was $1 \mathrm{~m}$, which was measured from the motor shaft to the tag's receiver.

The anchors were displaced at 2, 2 and $2 \mathrm{~m}$ along the axes X, Y, Z. First, the arm was moved into a starting position, which was considered to be 0 degrees. Then, the servo motor was rotating with a speed of 30 degrees / second. Results-wise, a delay can be observed between the triangulation-based estimation respectively the Kalman filter-based estimation (Figure 13). This delay explains the increase of variance of Kalman filter-based measurement compared with triangulation-based estimation.
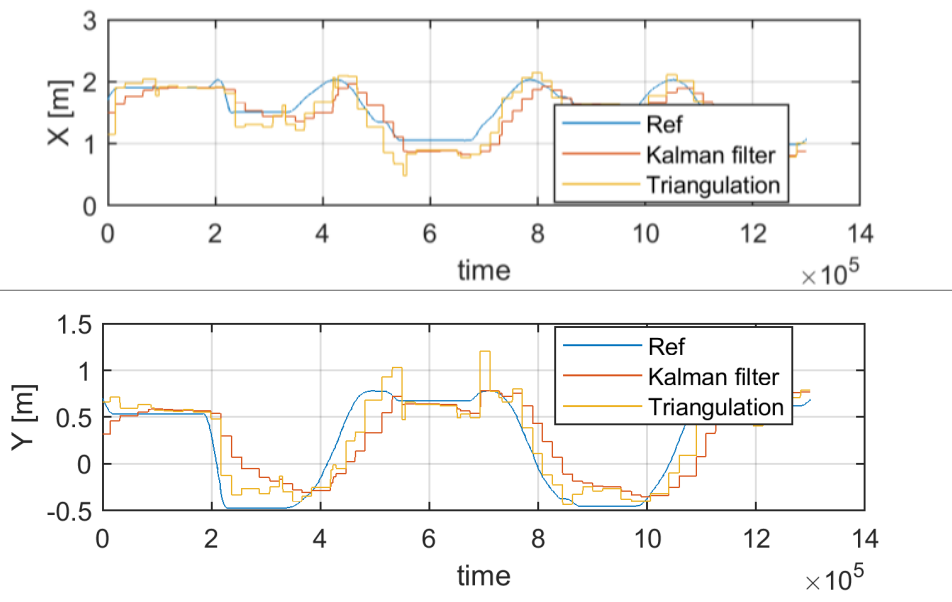


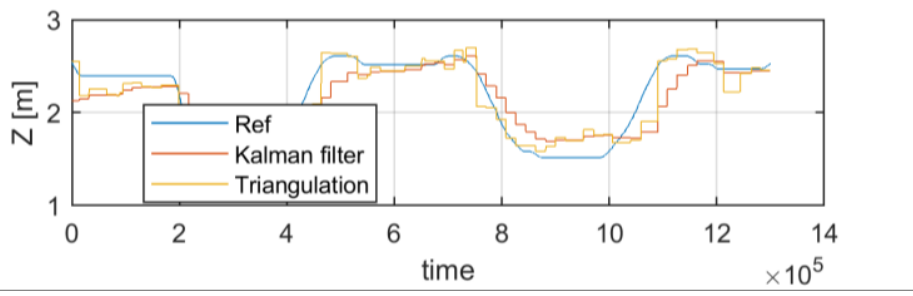

Figure 13

Real inputs, real processing of triangulation and Kalman filtering on FPGA for axes X, Y, Z. For each axis is presented the triangulation-based estimation (Triangulation), original reference signal (Ref), and results for Kalman filter
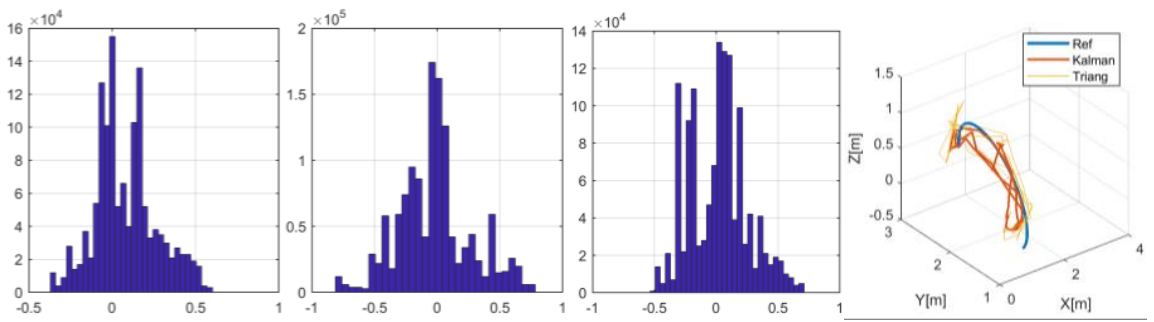

Figure 14

Error distribution along axes $\mathrm{X}, \mathrm{Y}$ and $\mathrm{Z}$ (from left to right) for Kalman filter-based estimation. Real measurement-based representation in XYZ coordinate system of the path evolution for original reference signal (Ref) and for Kalman filter (Kalman) and triangulation-based estimation (Triang).

The variances for triangulation are $0.0365,0.047$ and 0.0313 respectively for Kalman filter-based estimation $0.0347,0.0863$ and 0.056 .

For reduction of delay and respectively the variance, the increase of values used in the covariance matrix of measurement noise is proposed as resulted from simulations.

The error distribution for Kalman filter-based estimation along the three axes is presented in Figure 14 a) b) c). In tridimensional space the measurements with reference point coordinate, triangulation respectively Kalman filter-based coordinate estimation in Figure $14 \mathrm{~d}$ ) are presented.

\section{Conclusions}

The triangulation module for localization and the Kalman filter for noise reduction, has been successfully implemented in the FPGA and tuned. In System Generator a hardware co-simulation model was used to perform the measurements.

The developed localization model makes it possible to use both virtual inputs generated from Simulink for testing scopes and to receive real distance measurements from ultra-wide band RF modules. HLS and hardware co- 
simulation have greatly helped in the system's modules deployment, providing for different levels of test capabilities.

Instead of using frontend (RF modules), other solutions for distance measurement can also be used, the presented FPGA based localization solution is also applicable, the distances to the anchor can be introduced on the same serial interface.

There were some problems encountered during the FPGA based localization system implementation. One of the problems occurred during the export of the RTL model obtained (as a result of Vivado HLS synthesis) to the System Generator for hardware co-simulations.

Although both HLS simulation and synthesis were successfully completed, the module exported from Vivado HLS 2016.2 did not work in System Generator, the solution was an upgrade to Vivado HLS 2017.4. There were also compatibility concerns between the Vivado environment and the Matlab programs.

The simulations succeeded in verifying the correct operation of all the modules implemented in the FPGA. The IP cores generated from triangulation and Kalman filter modules can be integrated into mobile robot and UAV systems Zynq 7000 SoC family-based, control units, available in the University laboratory. Attention must be paid to the robustness of the RF ultra-wide band distance measurement unit, to increase the measurement sampling time and to minimize the loss of communication between RF modules.

\section{References}

[1] T. Haidegger, et al. "Controller design solutions for long distance telesurgical applications," International Journal of Artificial Intelligence 6.11 S, pp. 48-71, 2011

[2] S. Blažič, "On periodic control laws for mobile robots," IEEE transactions on industrial electronics 61.7, pp. 3660-3670, 2013

[3] K. Witrisal, P. Meissner, E. Leitinger, Y. Shen, C., Gustafson, F. Tufvesson, ... \& M. Z. Win, High-accuracy localization for assisted living: 5G systems will turn multipath channels from foe to friend. IEEE Signal Processing Magazine, 33(2), 59-70, 2016

[4] S. Palipana, B. Pietropaoli and D. Pesch, "Recent advances in RF-based passive device-free localisation for indoor applications," Ad Hoc Networks 64, pp. 80-98, 2017

[5] M. Ibrahim and O. Moselhi, "Inertial measurement unit based indoor localization for construction applications," Automation in Construction 71, pp. 13-20, 2016 
[6] R. F. Brena, J. P. García-Vázquez, C. E. Galván-Tejada, D. MuñozRodriguez, C. Vargas-Rosales, and J. Fangmeyer, "Evolution of indoor positioning technologies: A survey," Journal of Sensors 2017, 2017

[7] B. Van Herbruggen, et al., "Wi-PoS: A Low-Cost, Open Source UltraWideband (UWB) Hardware Platform with Long Range Sub-GHz Backbone," Sensors 19.7, pp. 1548, 2019

[8] G. Shi, and Y. Ming, "Survey of indoor positioning systems based on ultrawideband (UWB) technology," Wireless Communications, Networking and Applications. Springer, New Delhi, pp. 1269-1278, 2016

[9] S. Ullah, M. Ali, A. Hussain, and K. S. Kwak, "Applications of UWB technology" arXiv preprint arXiv:0911.1681, 2009

[10] L. Gogolak, S. Pletl, and D. Kukolj, "Neural network-based indoor localization in WSN environments," Acta Polytechnica Hungarica 10.6, pp. 221-235, 2013

[11] J. Simon, \& G. Martinovic, "Navigation of Mobile Robots Using WSN's RSSI Parameter and Potential Field Method". Acta Polytechnica Hungarica, 10(4), 107-118, 2013

[12] Y. Jiang, and V. C. Leung, "An asymmetric double sided two-way ranging for crystal offset," 2007 International Symposium on Signals, Systems and Electronics. IEEE, pp. 525-528, 2007

[13] I. Zs. Székely, \& S. T. Brassai, "Quadcopter control implemented on FPGA," 2019 IEEE $19^{\text {th }}$ International Symposium on Computational Intelligence and Informatics and $7^{\text {th }}$ IEEE International Conference on Recent Achievements in Mechatronics, Automation, Computer Sciences and Robotics (CINTI-MACRo) IEEE, 2019, pp. 49-54

[14] S. Hajdu, S. T. Brassai, I. Szekely, FPGA based angular stabilization of a quadcopter, MACRo 2015 Proceedings of the $5^{\text {th }}$ International Conference on Recent Achievements in Mechatronics, Automation, Computer Sciences and Robotics, 2(1), 79-86, 2017

[15] S. T. Brassai, B. Iantovics, C. Enachescu, "Optimization of robotic mobile agent navigation". Studies in Informatics and Control, 21(4), 403-412, 2012

[16] S. T. Brassai, B. Iantovics, C. Enăchescu, Artificial Intelligence in the path planning optimization of mobile agent navigation. Procedia Economics and Finance, 3, 243-250, 2012

[17] S. T. Brassai, C. Enăchescu, L. Losonczi, L. F. Márton, FPGA based embedded support for mobile robot sonar based navigation. In $201216^{\text {th }}$ International Conference on System Theory, Control and Computing (ICSTCC) (pp. 1-6) IEEE 2012 
[18] F. Zafari, A. Gkelias, and K. K. Leung, "A survey of indoor localization systems and technologies," IEEE Communications Surveys \& Tutorials 21.3, pp. 2568-2599, 2019

[19] B. Großwindhager, M. Stocker, M. Rath, C. A. Boano, and K. Römer, "SnapLoc: an ultra-fast UWB-based indoor localization system for an unlimited number of tags" $18^{\text {th }}$ ACM/IEEE International Conference on Information Processing in Sensor Networks (IPSN). IEEE, pp. 61-72, 2019

[20] V. Pierlot, and M. Van Droogenbroeck, "A new three object triangulation algorithm for mobile robot positioning" IEEE Transactions on Robotics 30.3, pp. 566-577, 2014

[21] U. Bekcibasi, and M. Tenruh, "Increasing RSSI localization accuracy with distance reference anchor in wireless sensor networks," Acta Polytechnica Hungarica 11.8, pp. 103-120, 2014

[22] M. Kwak, and J. Chong, "A new double two-way ranging algorithm for ranging system," $20102^{\text {nd }}$ IEEE InternationalConference on Network Infrastructure and Digital Content. IEEE, pp. 470-473, 2010

[23] https://www.decawave.com/news/current-news/decawave-claims-accuracywithin-centimeters-indoor-positioning-chip-fiercewireless

[24] M. Varshosaz, H. Helali, and D. Shojaee, "The methods of triangulation," Map Middle East'05: Proceedings of the $1^{\text {st }}$ Annual Middle East Conference and Exhibition on Geospatial Information, Technology and Applications, 2005

[25] A. Küpper, "Location-based services: fundamentals and operation,” John Wiley \& Sons, 2005

[26] Y. Kim, and H,"Bang, "Introduction to Kalman Filter and Its Applications," Introduction and Implementations of the Kalman Filter. IntechOpen, 2018 